Lessons I ear ned from twent y-year oper at i on of the Large Hel i cal Devi ce pol oi dal coi I s made from cabl e- i n-conduit conduct or s

\begin{tabular}{|l|l|}
\hline $\begin{array}{l}\text { j our nal or } \\
\text { publ i cat } \mathrm{i} \text { on } \mathrm{titl} \text { e }\end{array}$ & Cryogeni cs \\
\hline vol une & 91 \\
\hline page $\mathrm{r}$ ange & $1-6$ \\
\hline year & $2018-02-06$ \\
\hline NAl S & 11202 \\
\hline URL & ht t p: //hdl . handl e. net /10655/00012610 \\
\hline
\end{tabular}




\title{
Lessons learned from twenty-year operation of the Large Helical Device poloidal coils made from cable-in-conduit conductors
}

Kazuya Takahata, b, *, Sadatomo Moriuchi ${ }^{a}$, Kouki Ooba ${ }^{a}$, Shigeyuki Takami ${ }^{a}$, Akifumi Iwamoto ${ }^{a}$, Toshiyuki Mito ${ }^{\mathrm{a}, \mathrm{b}}$ and Shinsaku Imagawa, $\mathrm{a}^{\mathrm{a}} \mathrm{b}$

National Institute for Fusion Science ${ }^{\mathrm{a}}$, National Institutes of Natural Sciences, 322-6 Oroshi, Toki, Gifu, 509-5292 Japan

SOKENDAI (The Graduate University for Advanced Studies) ${ }^{\mathrm{b}}$, 322-6 Oroshi, Toki, Gifu, 509-5292 Japan

*Corresponding author.

E-mail address: takahata@nifs.ac.jp (K. Takahata).

\begin{abstract}
The Large Helical Device (LHD) superconducting magnet system consists of two pairs of helical coils and three pairs of poloidal coils. The poloidal coils use cable-in-conduit (CIC) conductors, which have now been adopted in many fusion devices, with forced cooling by supercritical helium. The poloidal coils were first energized with the helical coils on March 27, 1998. Since that time, the coils have experienced 54,600 h of steady cooling, 10,600 h of excitation operation, and nineteen thermal cycles for twenty years. During this period, no superconducting-tonormal transition of the conductors has been observed. The stable operation of the poloidal coils demonstrates that a CIC conductor is suited to large-scale superconducting magnets. The AC loss has remained constant, even though a slight decrease was observed in the early phase of operation. The hydraulic characteristics have been maintained without obstruction over the entire period of steady cooling. The experience gained from twenty years of operation has also provided lessons regarding malfunctions of peripheral equipment.
\end{abstract}

\section{Keywords}

cable-in-conduit (CIC) conductor

AC loss

hydraulic characteristics

quench detection system

cryogenic insulation break

\section{Introduction}

The Large Helical Device (LHD) superconducting magnet system consists of two pairs of helical coils and three pairs of poloidal coils [1]. The helical and poloidal coils use Nb-Ti composite conductors with pool cooling by liquid helium at $3.2 \mathrm{~K}$, and cable-in-conduit (CIC) conductors with forced cooling by supercritical helium at $4.4 \mathrm{~K}$, respectively. To our knowledge, this is the first time that a CIC conductor has been applied to a magnet for a large-scale fusion device for creating plasmas.

The poloidal coil system consists of three pairs of circular solenoids called the inner vertical (IV) coils, inner shaping (IS) coils, and outer vertical (OV) coils (Fig. 1). The main parameters of the poloidal coils are listed in Table 1. To distinguish the lower and upper coils, the letters "-L" and "-U" are added to the abbreviations. For example, the upper IV coil is referred to as IV-U coil. The coils are formed from continuous conductors wound into two-layer coils, which are referred to as a double-pancake, and are stacked as eight double-pancakes, molded, and electrically connected in series. The conductors are 
CIC type with $486 \mathrm{Nb}-\mathrm{Ti} / \mathrm{Cu}$ composite wires. The surface of the wires is bare without any coating. The strands are twisted in five stages to produce a cable. The cable is then covered with a rectangular conduit made of stainless steel.

The poloidal coils have successfully contributed to plasma experiments for twenty years, together with the helical coils. In this paper, we describe long-term changes in the electromagnetic and hydraulic characteristics over these twenty years. Furthermore, the experiences gained from long-term operation have provided several lessons for large-scale superconducting magnet systems. Here we introduce these lessons and present the countermeasures taken to preclude future issues.

\section{Operational history}

The poloidal coils were first energized with the helical coils on March 27, 1998 [2]. The successful manufacturing and initial stable operation of the poloidal coils demonstrated that a CIC conductor is better suited to large-scale superconducting magnets than other conductor designs. The coils have since experienced 54,600 h of steady cooling, 10,600 h of excitation operation, and nineteen thermal cycles (Fig. 2). A series of continuous cooling operations is referred to as a campaign, and thus the LHD has experienced nineteen campaigns. The number of excitations has reached 1,749. During the entire period, no superconducting-to-normal transition of the conductors has been observed. The current, maximum magnetic field, and temperature margins when operating at the highest load factor with various operational conditions are listed in Table 2. These data demonstrate that superconducting coils with $\mathrm{Nb}$ Ti CIC conductors can be stably operated over a long period if the temperature margin is at least $2.4 \mathrm{~K}$.

In 2008, additional pulse power supplies for the IV and IS coils were installed to enhance the output voltage of the power supplies from $33 \mathrm{~V}$ to $213 \mathrm{~V}$ [3]. The maximum rate of field change increased to $0.06 \mathrm{~T} / \mathrm{s}$, which corresponds to a six-fold increase over previous operations. The pulse operation of the poloidal coils was applied to magnetic axis swing experiments of the LHD, and the poloidal coils operated stably, even though false detection events of the quench detection system have interrupted experiments several times, as described in Section 5.

\section{Long-term monitoring of AC loss}

Long-term monitoring of the electromagnetic and hydraulic characteristics of the coils has been performed. First, AC loss was monitored by measurement of the inlet and outlet coolant temperatures during discharge of the coils after daily plasma experiments [3]. Fig. 3 shows the variation in AC loss from the OV and IV coils over the period from the third to the nineteenth campaigns. The plots indicate the average loss at several discharges from the same excitation conditions (Table 3). The sweep time $\Delta T$ was set to 1,650 s during the third and the early part of the fourth campaigns, and was changed to $825 \mathrm{~s}$ after the latter part of the fourth campaign. In the first and second campaigns during the first year, no excitation with the same conditions was performed. Thus, there are no data for this period. The data for the IS coils are also omitted because the power loss is much lower than that for the OV and IV coils, and sufficient measurement accuracy was not obtained. The calculation shows that the loss may include a hysteresis loss of $10.6 \mathrm{~kJ}$ and $7.5 \mathrm{~kJ}$ for the OV and IV coils, respectively [4]. The remaining loss corresponds to interfilament and interstrand coupling losses. We have previously reported that the interstrand coupling loss dominated the total coupling loss of the conductors in the LHD poloidal coils [5].

Even though the AC loss decreased slightly until the fifth campaign, except for the IV-L coil, the losses have since remained unchanged. The initial change was probably caused by a decrease in an interstrand coupling loss due to electrical contact between strands. The remaining hysteresis and interfilament coupling losses are unlikely to vary because they are dependent only on the structure of the strand. Electromagnetic forces during excitation may move the strands and break electrical contacts between them. Such contacts can be produced by plastic deformation of the strand surface during the 
cabling and jacketing processes. The maximum electromagnetic force did increase gradually during trials aimed at high-field operation, and peaked in the fourth campaign. The maximum Lorentz forces acting on the cable in the IV and OV coils are approximately 83 and $67 \mathrm{kN} / \mathrm{m}$, respectively. We were initially concerned that the $\mathrm{AC}$ loss may increase with the excitation number, as reported previously $[6,7]$. However, our results demonstrate that there was no tendency for it to increase over a long period. The increase reported previously was probably due to the influence of the coating on the strand surface, which is not applied to the LHD poloidal coils, and larger Lorentz forces.

\section{Long-term monitoring of the friction factor}

An important issue for stable operation of forced-cooled coils is to maintain the hydraulic characteristics for a long period. Coolant pressure drops were thus monitored by measuring the inlet and outlet pressures during steady cooling [8]. Fig. 4 shows the variation in the average friction factor over each campaign period for the six coils. There was little difference in the Reynolds number for each campaign. The friction factor is dependent on the Reynolds number. Therefore, the average value can be extrapolated to the value at a Reynolds number of 3,000, according to the empirical relationship proposed by Katheder [9]. The friction factor showed a tendency to decrease over the campaigns. We suggest that this was due to cleaning of the strand surfaces and the narrow cooling channels by the forced-flow helium coolant. The sudden increase in the friction factor in the fifteenth campaign, which had no effect on operation, was probably caused by tiny particles of solidified gasses in the helium coolant. The impurity gases are considered to originate from the compressor oil of the helium refrigerator, which was changed just before the fifteenth campaign. However, the friction factor decreased at the sixteenth campaign and subsequently maintained a low level similar to that before the fourteenth campaign.

The poloidal coils have two mesh filters with a mesh size of $5 \mu \mathrm{m}$ at the helium coolant inlet to remove solidified gas and vapor. The filters can be switched when one of the two is clogged. The clogged filter can be warmed up independently and evacuated to clear impurities. The mesh filters did become clogged during cool-down in the first to the fourth campaigns and during the fifteenth campaign. The friction factor during steady-cooling tended to be higher after clogging of the filters during cool-down. This further confirms that impurity gasses affected the friction factor. In addition, the filters probably played an important role in the removal of the impurity gasses. The results demonstrate that the hydraulic characteristics can be maintained without obstruction over a long period of steady cooling if careful attention is paid to impurity gases in helium.

\section{Malfunction and false detection events of the quench detection system}

Operation of the superconducting magnet system must always remain stable. However, a malfunction of peripheral equipment can stop continuous operation. Even the poloidal coil system has experienced events that have interrupted plasma experiments due to malfunctions. Here we introduce these malfunctions, specifically false detection events of the quench detection system and a helium leak from a cryogenic insulation break.

The quench detection system forces the coils into fast discharge to protect the conductors from excessive temperature increase due to a superconducting-to-normal transition. However, fast discharge involves the risk of electrical breakdown in the coil. Therefore, an unnecessary discharge due to malfunction should be avoided. Table 4 lists the history of malfunctions and false detection events experienced by the quench detection system for the poloidal coils. The system can send out signals that demand slow (a time constant of $300 \mathrm{~s}$ ) or fast (30 s) discharges, which correspond to the detected burst voltage duration. The voltage threshold was set to $0.2 \mathrm{~V}$ in each case. The criterion for burst duration was set initially to $1 \mathrm{~s}$ and $3 \mathrm{~s}$ for the slow and fast discharges, respectively. The criterion for burst duration plays a role in the elimination of false detection events due to unexpected voltage spikes. A detection delay of $3 \mathrm{~s}$ for the fast discharge is the upper permissible limit determined by a temperature 
rise during a quench, which is limited to $250 \mathrm{~K}$. Therefore, the criterion for the fast discharge should remain unchanged. In practice, when the excitation is turned off and the demand signal is sent out the coils are not discharged.

In 2004, the system malfunctioned frequently and synchronously with the neutral beam injection (NBI) pulse during conditioning (\#2). At that time, the coils were not excited. The surge from the NBI probably exceeded the threshold level for detection of an instrument fault (for example, $11 \mathrm{~V}$ ), such as breaking of a wire. A criterion for voltage pulse duration was then added to the instrument fault detection. The malfunction stopped once, but subsequently occurred frequently again. The next malfunction (\#3) was caused by a surge into external wiring for remote operation. The function to control the detector remotely was then removed. The malfunction related to the NBI was finally avoided by these measures in advance of subsequent plasma experiments.

The slow discharges in 2005 (\#6) and 2008 (\#8) were caused by the coupling current, which is an intrinsic property of a composite superconductor including a CIC conductor, during magnetic axis swing experiments [10]. Fig. 5 shows the out-of-balance voltage between the upper and lower IS coils, which compensates for the inductive terminal voltage using a balanced bridge circuit [11]. At that time, the current in the IS coils decreased from $339 \mathrm{~A}$ to $218 \mathrm{~A}$ for $2 \mathrm{~s}$. Voltage spikes with a peak of $0.6 \mathrm{~V}$ were observed just after the start and end of the swing, even though the coils were fully superconductive. The anomalous spikes were caused by the voltage due to the coupling current, which was not perfectly compensated by the bridge circuit [11]. In addition, the difference in the time constants of the coupling current between the two symmetric coils may have also had an effect. The plasma also induced an additional voltage, and the out-of-balance voltage then exceeded the quench detection threshold $(0.2 \mathrm{~V})$ for almost $1 \mathrm{~s}$, which is the duration criterion. The slow discharges were triggered by the effect of both the swing and the plasma. In this case, the quench detection system itself functioned normally. Theoretical analyses indicated that the duration of the voltage spike is weakly dependent on the magnetic field and sweep rate, and is strongly dependent on the time constant of the coupling currents [11]. This dependency was also confirmed experimentally. For example, Fig. 6 shows the out-of-balance voltage during magnetic axis swings for $1 \mathrm{~s}$ and $2 \mathrm{~s}$. The data for $2 \mathrm{~s}$ is the same as those in Fig. 5. Although the peak voltage increased twofold in proportion to the sweep rate, such an increase in the duration of the voltage spike was not observed. The period during which the voltage exceeded the criterion of $0.2 \mathrm{~V}$ was increased by $36 \%$. Therefore, it was decided to increase the duration criterion from $1 \mathrm{~s}$ to $1.5 \mathrm{~s}$. This change enabled unnecessary discharges due to the coupling current to be avoided.

The malfunction in 2017 (\#10) resulted in fast discharge during excitation for the first time. However, the new detector that replaced one of the six detectors on trial was faulty. The preventive maintenance finished with a poor result. We realized that preliminary operation checks of the new detector may have been insufficient.

\section{Helium leak from cryogenic insulation break}

A cryogenic insulation break is an essential component for CIC conductors because both current and coolant flow through the conductor. The poloidal coil system has 120 cryogenic insulating breaks made of fiber-reinforced plastic (FRP). During the sixteenth cool-down of the coils in 2012, one of the breaks suddenly leaked helium due to cracking of a plastic adhesive material between the FRP and the stainlesssteel pipes. The adhesive cracked simultaneously at both ends of the FRP pipe, as shown in Fig. 7. This was unexpected, because all the breaks were cycled in the temperature range from room temperature to $77 \mathrm{~K}$ ten times for quality assurance before installation on the LHD, and because the break has a flexible corrugated pipe at one side to absorb thermal stress (right side in the figure). Thus, it is unlikely that only thermal stress caused the cracking. Further investigation is required to clarify the age degradation and creep behavior of the plastic adhesive materials [12].

All of the breaks were replaced with new improved breaks after the sixteenth experimental campaign. In the former break, an FRP pipe was inserted into a stainless steel pipe and the two pipes were adhered together. In the new break, an additional outer FRP pipe was covered and adhered to the outside surface 
of the stainless steel and the inner FRP pipes, as shown in Fig. 8. The adhesive area was thus increased by approximately $70 \%$. A sudden helium leak carries a risk of electrical breakdown. Thus, insulation breaks should be designed conservatively for future devices.

\section{Conclusion}

The LHD poloidal coils have been operated stably for twenty years and nineteen campaigns. Longterm monitoring of the electromagnetic and hydraulic characteristics of the coils has been performed. Even though the AC loss slightly decreased until the fifth campaign, the losses have since remained unchanged. The friction factor showed a tendency to decrease over the campaigns, except for an increase in the fifteenth campaign, which was considered to be induced by solidified impurity gases from the oil compressor, although this was still acceptable and had no effect on stable operation. For future superconducting magnet systems, long-term monitoring of the electromagnetic and hydraulic characteristics will be necessary in order to provide dependable operation.

Even though the coils themselves have been electromagnetically and hydraulically stable, malfunctions of the peripheral equipment have interrupted plasma experiments. The quench detection system malfunctioned or generated false detection events ten times over the campaigns. The false detection events were caused by out-of-balance voltages due to coupling currents, which is an intrinsic property of a composite superconductor. This phenomenon should have been realized before operation with a change of magnetic field. The malfunctions should also have been avoided by a stricter regimen of preliminary operation checks. In the sixteenth campaign, one of the 120 cryogenic insulation breaks suddenly leaked helium, due to cracking of the plastic adhesive material. The cryogenic insulation break should have been designed to have a larger adhesive area. These lessons learned will help in the design and maintenance of future superconducting magnet systems.

\section{Acknowledgments}

This work was performed with the support and under the auspices of the National Institute for Fusion Science (NIFS) Collaborative Research Program. (NIFS11ULAA703). The authors wish to thank the members of the Department of Engineering and Technical Services of NIFS for invaluable technical assistance, and Toshiba Corporation for periodic inspections and repairs. This paper is dedicated to the late J. Yamamoto, who initiated research and development of the superconducting coils for the LHD.

\section{References}

[1] Fujiwara M, Yamazaki K, Okamoto M, Todoroki J, Amano T, Watanabe T, et al. Large helical device (LHD) program. J. Fusion Energy 1996;15:7-153.

[2] Iiyoshi A, Komori A, Ejiri A, Emoto M, Funaba H, Goto M, et al. Overview of the Large Helical Device project. Nucl. Fusion 1999;39:1245-56.

[3] Takahata K, Chikaraishi H, Mito T, Imagawa S. AC losses in poloidal coils of the Large Helical Device. IEEE Trans. Appl. Supercond. 2010;20:517-20.

[4] Takahata K, Chilaraishi H, Mito T, Imagawa S. Hysteresis loss in poloidal coils of the Large Helical Device. Plasma Fusion Res. 2011;6.2405077.

[5] Takahata K, Mito T, Chikaraishi H, Imagawa S, Satow T. Coupling losses in cable-in-conduit conductors for LHD poloidal coils. Fusion Eng. Des. 2003;65:39-45.

[6] Zanino R, Bagnasco M, Ciazynski D, Lacroix B, van Lanen EPA, Nicollet S, et al. EU contribution to the test and analysis of the ITER poloidal field conductor insert and the central solenoid model coil. Supercond. Sci. Technol. 2009;22:085006. 
[7] Nijhuis A, Ilyin Yu, Abbas W, ten Haken B, ten Kate HHJ. Change of interstrand contact resistance and coupling loss in various prototype ITER NbTi conductors with transverse loading in Twente Cryogenic Cable Press up to 40,000 cycles. Cryogenics 2004;44:319-339.

[8] Takahata K, Moriuchi S, Ooba K, Mito T, Imagawa S. Long-term monitoring of hydraulic characteristics of LHD poloidal coils. Plasma Fusion Res. 2012;7:2405008.

[9] Katheder H. Optimum thermohydraulic operation regime for cable in conduit superconductors (CICS). Cryogenics 1994;34:595-8.

[10] Sakakibara S, Ohdachi S, Watanabe KY, Suzuki Y, Funaba H, Narushima Y, et al. Exploration of optimal high-beta operation regime by magnetic axis swing in the Large Helical Device. Proc. 23rd Fusion Energy Conf. (Daejeon, Korea, 11-16 October 2010) EXS/P5-13.

[11] Takahata K, Imagawa S, Yanagi N, Chikaraishi H, Mito T. Influence of magnetic hysteresis on quench-voltage detection in large superconducting magnets. Fusion Eng. Des. 2006;81:2571-5.

[12] Costa I, Barros J. Tensile creep of a structural epoxy adhesive: Experimental and analytical characterization. International Journal of Adhesion \& Adhesives 2015;59:115-24.

\section{Figure Captions}

Fig. 1. LHD superconducting magnet system.

Fig. 2. Cumulative operating time for steady cooling and excitation.

Fig. 3. Interannual trends in AC losses from the OV and IV coils over the nineteen campaigns.

Fig. 4. Interannual trends in friction factor over the nineteen campaigns for the six LHD poloidal coils. The friction factor is extrapolated to that at a Reynolds number of 3,000.

Fig. 5. Out-of-balance voltage from the IS coils during magnetic axis swings for $2 \mathrm{~s}$, with and without plasma.

Fig. 6. Out-of-balance voltage from the IS coils during magnetic axis swings for $1 \mathrm{~s}$ (solid line) and $2 \mathrm{~s}$ (dashed line) without plasma.

Fig. 7. Soap bubble testing for the cryogenic insulation break after a helium leak. Bubbles are evident at both ends of the FRP pipe.

Fig. 8. New improved (upper) and former (lower) cryogenic insulation breaks. The new break has an additional outer FRP pipe. 
Table 1 Main parameters for the LHD poloidal coils.

\begin{tabular}{lccc}
\hline & OV & IS & IV \\
\hline Center diameter (m) & 11.1 & 5.6 & 3.6 \\
Height (m) & 0.54 & 0.46 & 0.46 \\
Weight $^{\mathrm{a}}$ (ton) & 45 & 25 & 16 \\
Number of turns $^{\mathrm{a}}$ & 144 & 208 & 240 \\
Inductance $^{\mathrm{a}}(\mathrm{H})$ & 0.52 & 0.45 & 0.31 \\
Conductor dimension $^{\left(\mathrm{mm}^{2}\right)}$ & $27.5 \times 31.8$ & $23.0 \times 27.6$ & $23.0 \times 27.6$ \\
\hline aPer coil & & &
\end{tabular}

Table 2 Current, magnetic field, and temperature margins when operating at the highest load factor.

\begin{tabular}{lccc}
\hline & OV & IS & IV \\
\hline Current (kA) & 20.6 & 16.2 & 15.6 \\
Magnetic field (T) & 3.29 & 3.44 & 5.34 \\
Temperature margin (K) & 3.1 & 3.0 & 2.4 \\
\hline
\end{tabular}

Table 3 Excitation conditions for measurement of the AC loss.

\begin{tabular}{ccc}
\hline & $\begin{array}{c}\text { Current } \\
(\mathrm{kA})\end{array}$ & $\begin{array}{c}\text { Maximum } \\
\text { field (T) }\end{array}$ \\
\hline Helical coils & 11.0 & \\
OV coils & 18.0 & 2.86 \\
IS coils & 3.0 & 1.44 \\
IV coils & 11.2 & 3.47
\end{tabular}


Table 4 History of malfunctions and false detection events of the quench detection system for the LHD poloidal coils.

\begin{tabular}{lllll}
\hline$\#$ & Demand signal & Excitation & Cause & Year/Month \\
\hline 1 & Slow discharge & ON & Malfunction of timer circuit & $2002 / 11$ \\
2 & $\begin{array}{l}\text { Fast discharge } \\
\text { (frequently) }\end{array}$ & OFF & Surge voltage from NBI conditioning & $2004 / 4$ \\
3 & $\begin{array}{l}\text { Fast discharge } \\
\text { (frequently) }\end{array}$ & OFF & Surge voltage from NBI conditioning & $2004 / 9$ \\
4 & Slow discharge & ON & Unknown & $2004 / 12$ \\
5 & Fast discharge & OFF & Unknown & $2005 / 6$ \\
6 & Slow discharge & ON & False detection during magnetic axis & $2005 / 11$ \\
7 & Slow discharge & ON & Malfunction of potentiometer & $2008 / 10$ \\
8 & Slow discharge (3 times) & ON & False detection during magnetic axis & $2008 / 10$ \\
9 & Fast discharge & OFF & Lightning surge & $2009 / 8$ \\
10 & Fast discharge & ON & Malfunction of electronic substrate & $2017 / 2$ \\
\hline
\end{tabular}




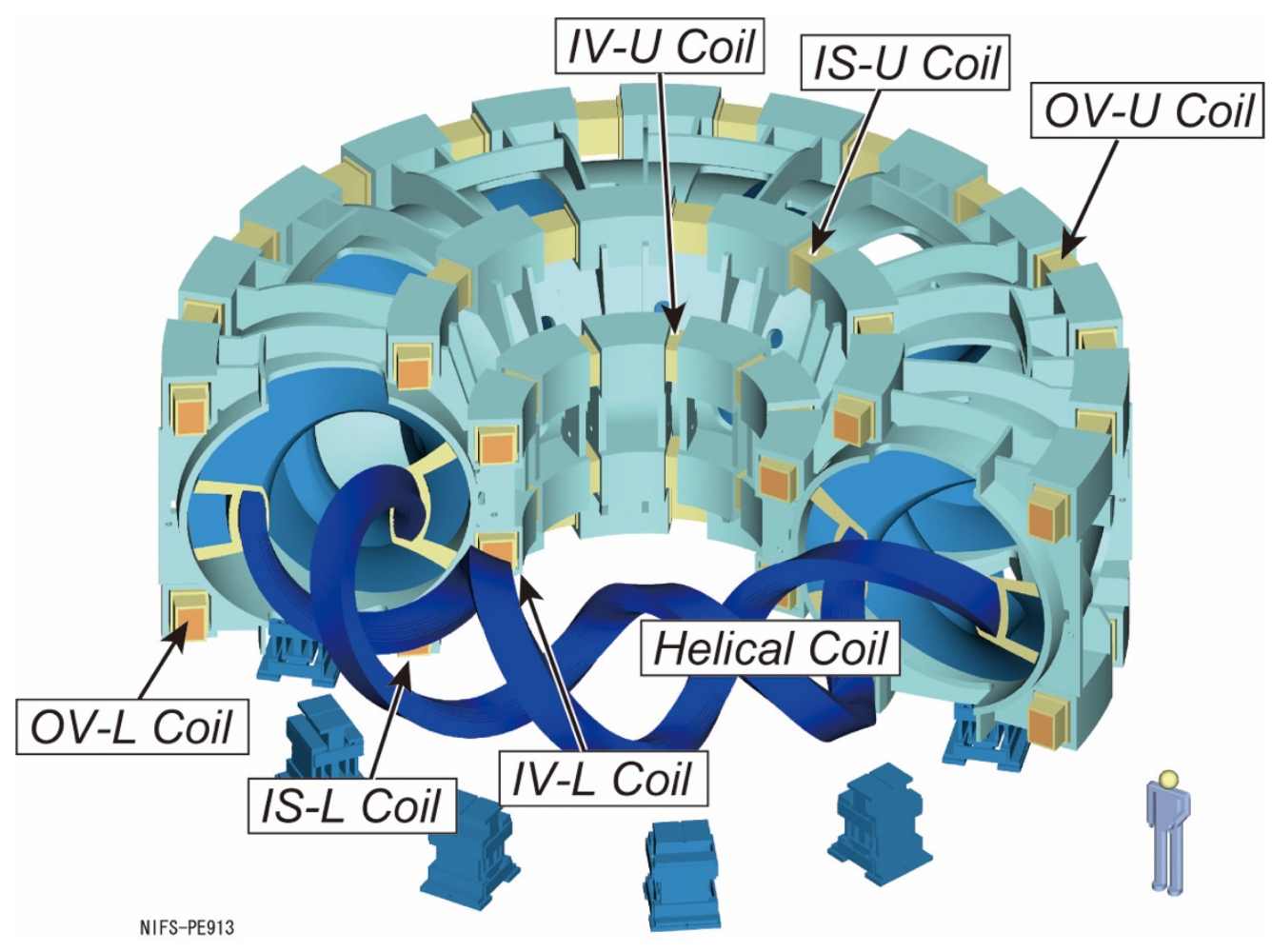

Fig. 1. LHD superconducting magnet system.

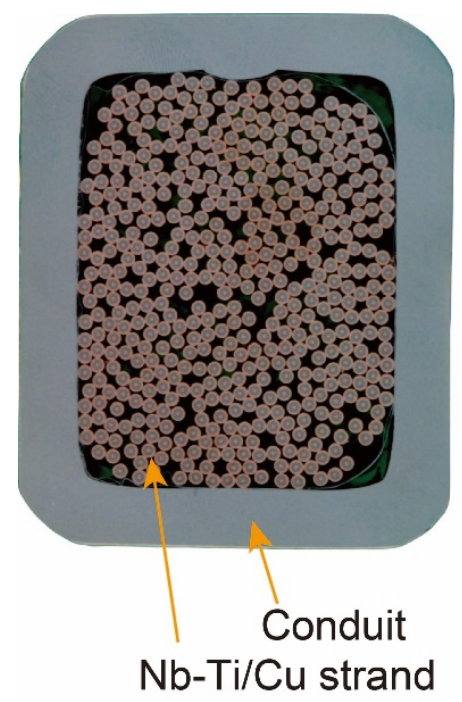

Fig. 2. Cross-sectional image of the CIC conductor for the IV coils. 


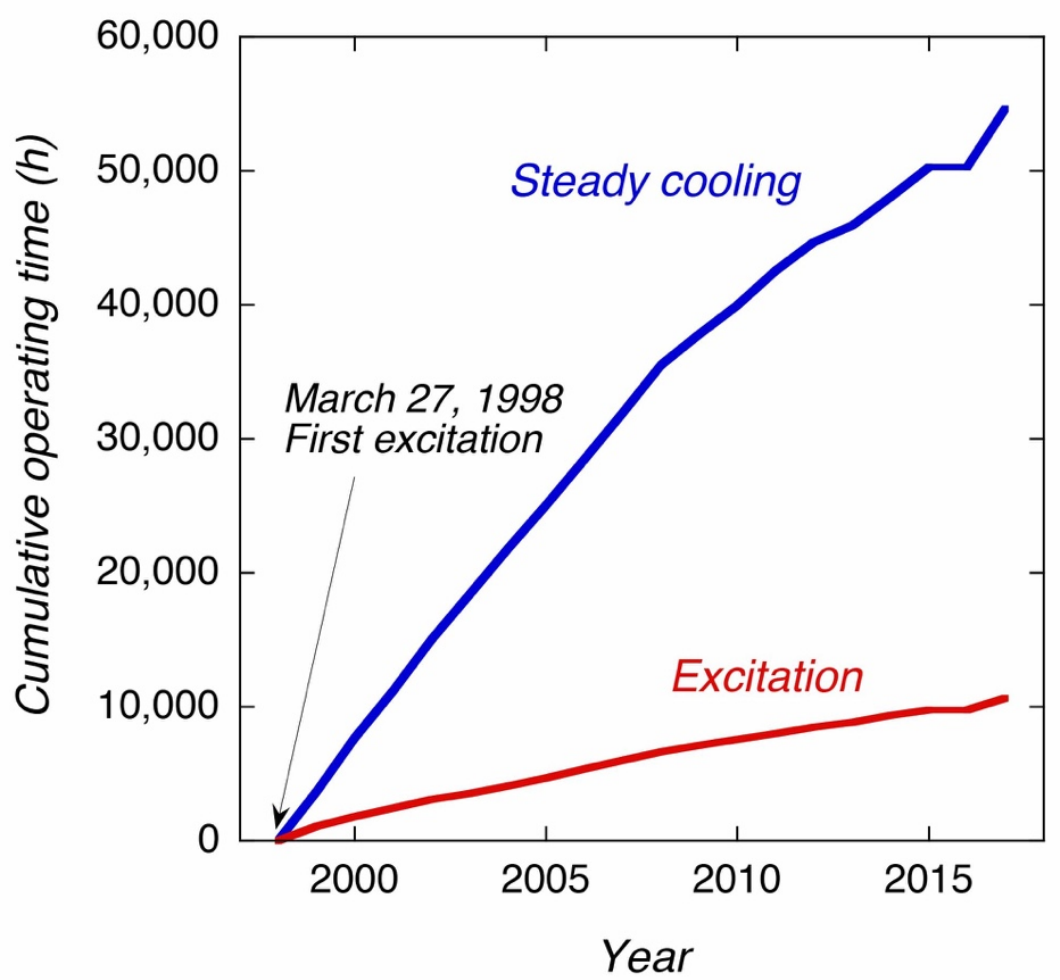

Fig. 3. Cumulative operating time for steady cooling and excitation.

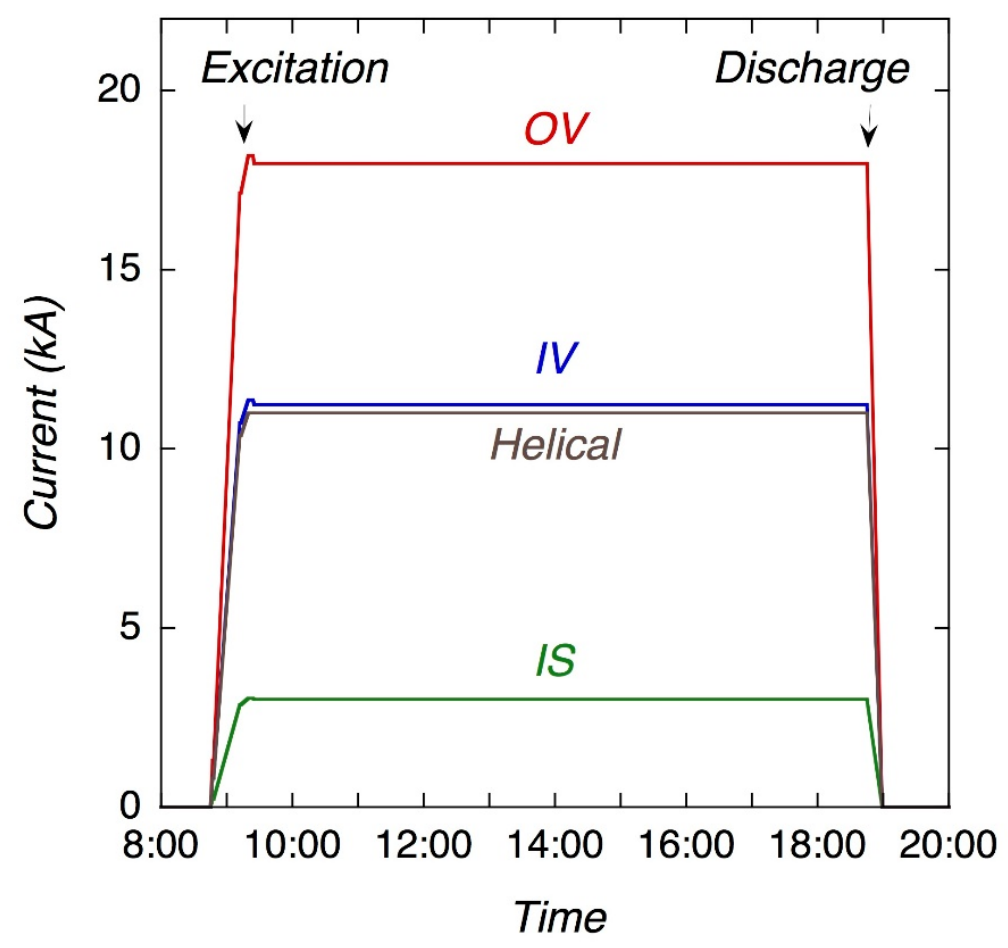

Fig. 4. Coil current waveform on March 3, 2017. 


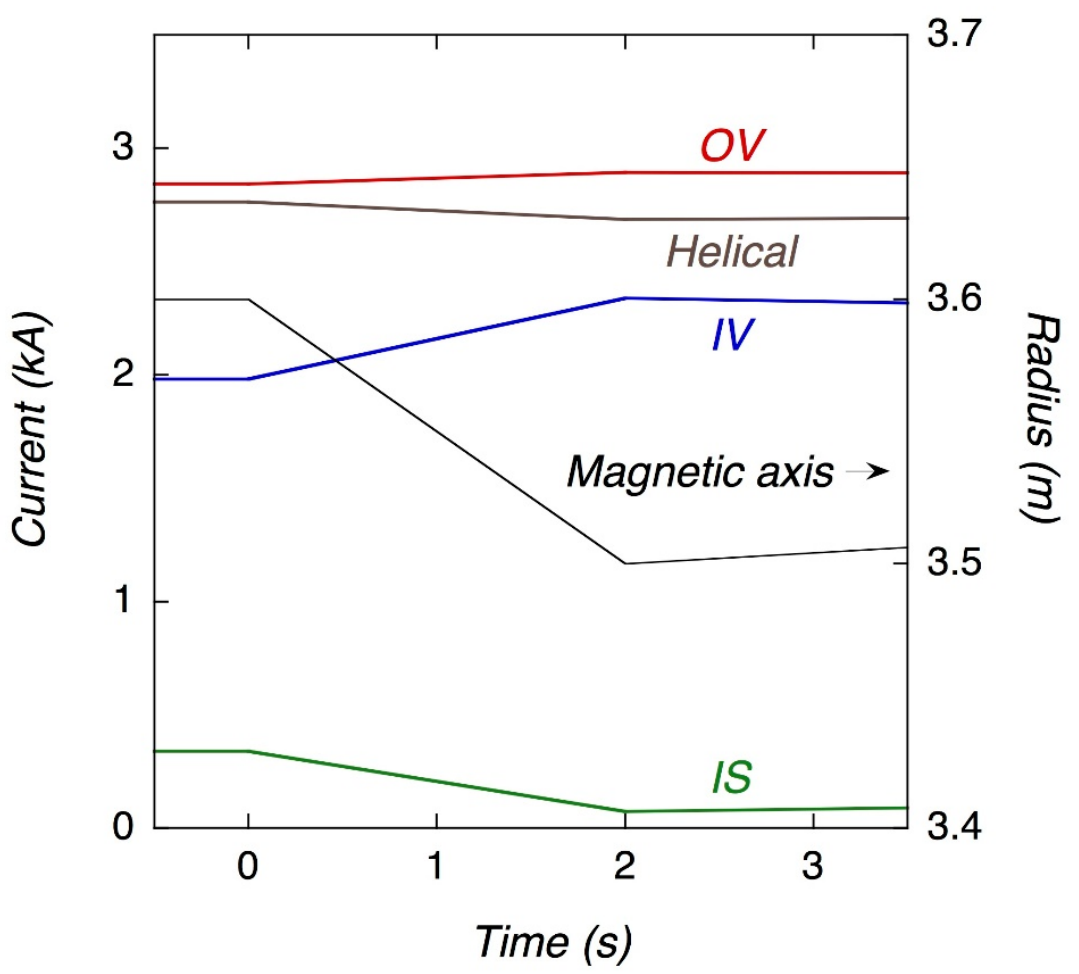

Fig. 5. Time evolution of the coil currents and the magnetic axis during the magnetic axis swing.

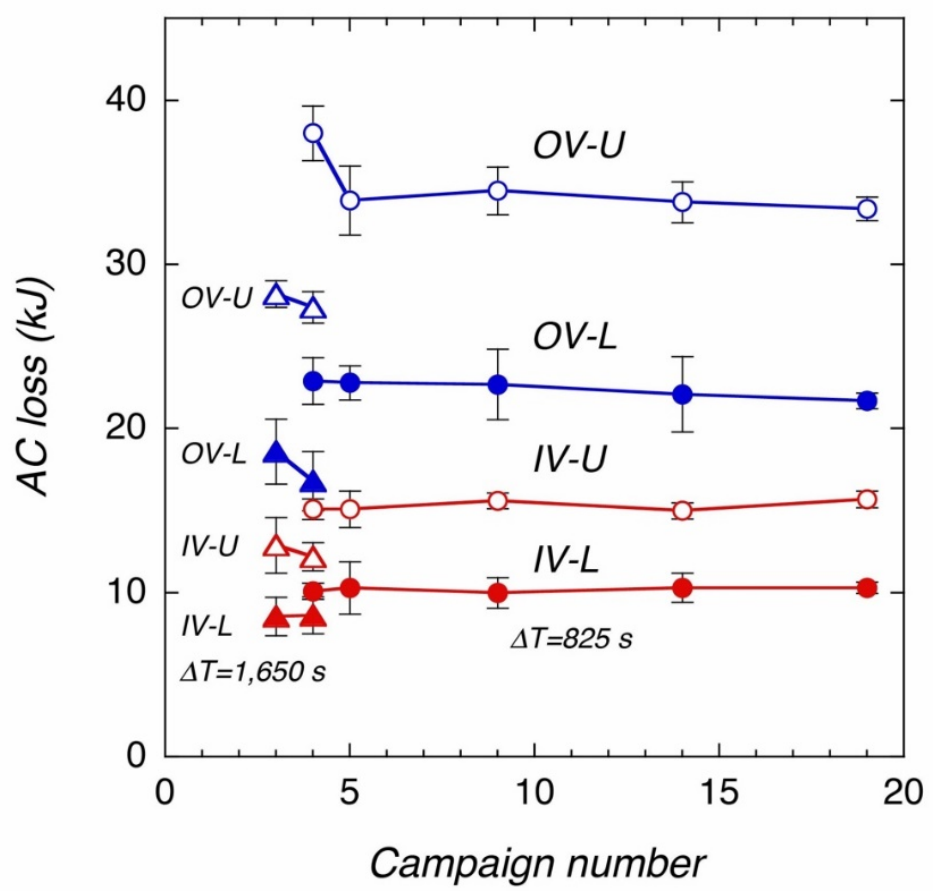

Fig. 6. Interannual trends in AC losses from the OV and IV coils over the nineteen campaigns. 


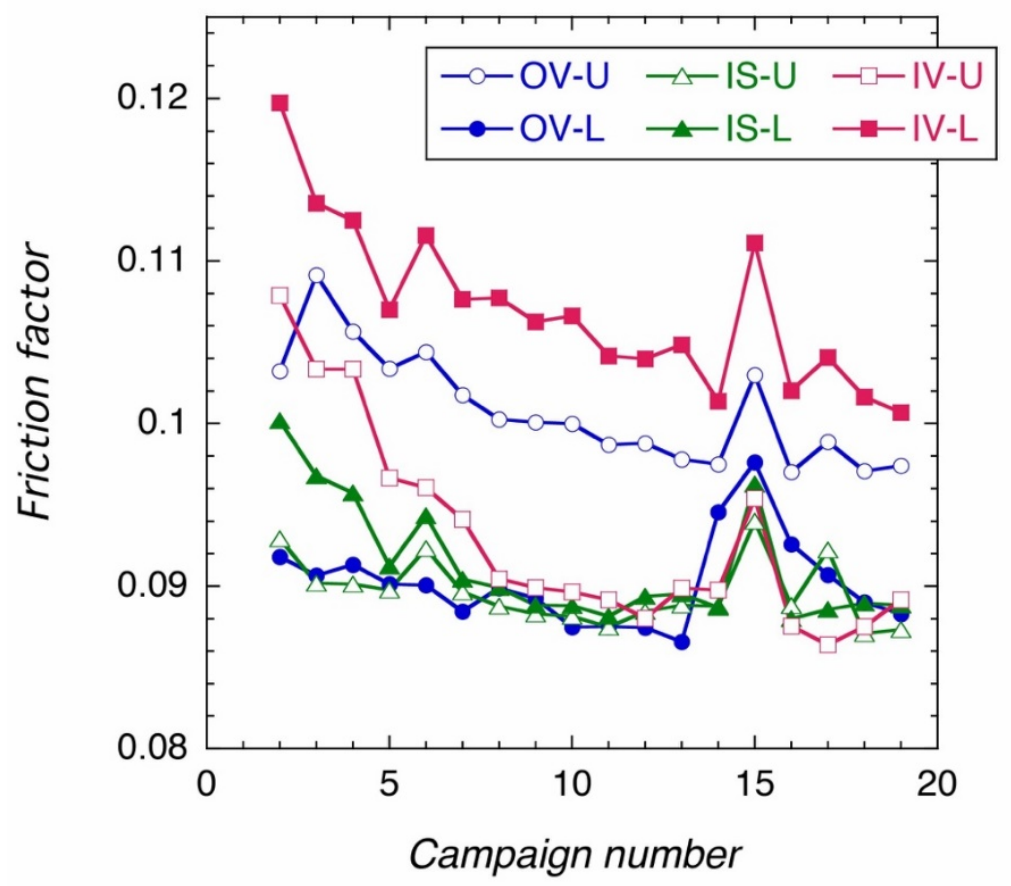

Fig. 7. Interannual trends in friction factor over the nineteen campaigns for the six LHD poloidal coils. The friction factor is extrapolated to that at a Reynolds number of 3,000.

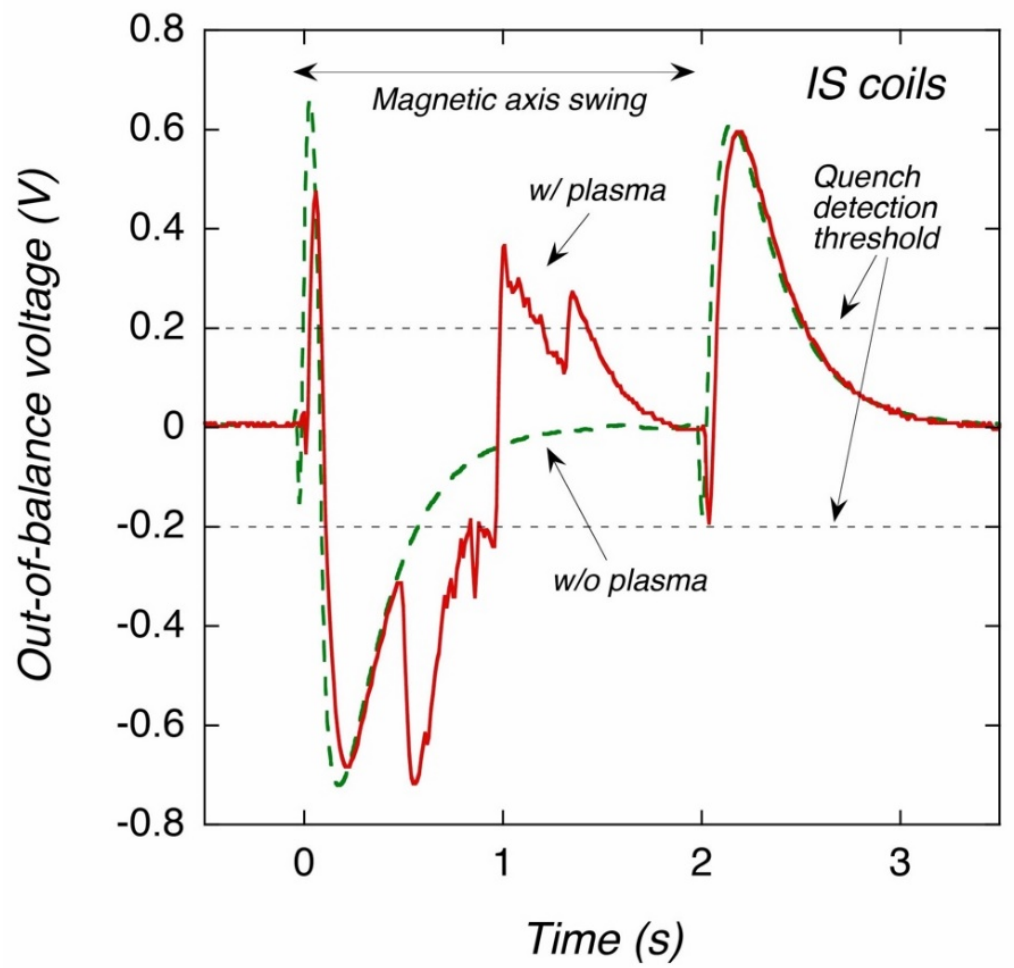

Fig. 8. Out-of-balance voltage from the IS coils during magnetic axis swings for $2 \mathrm{~s}$, with and without plasma. 


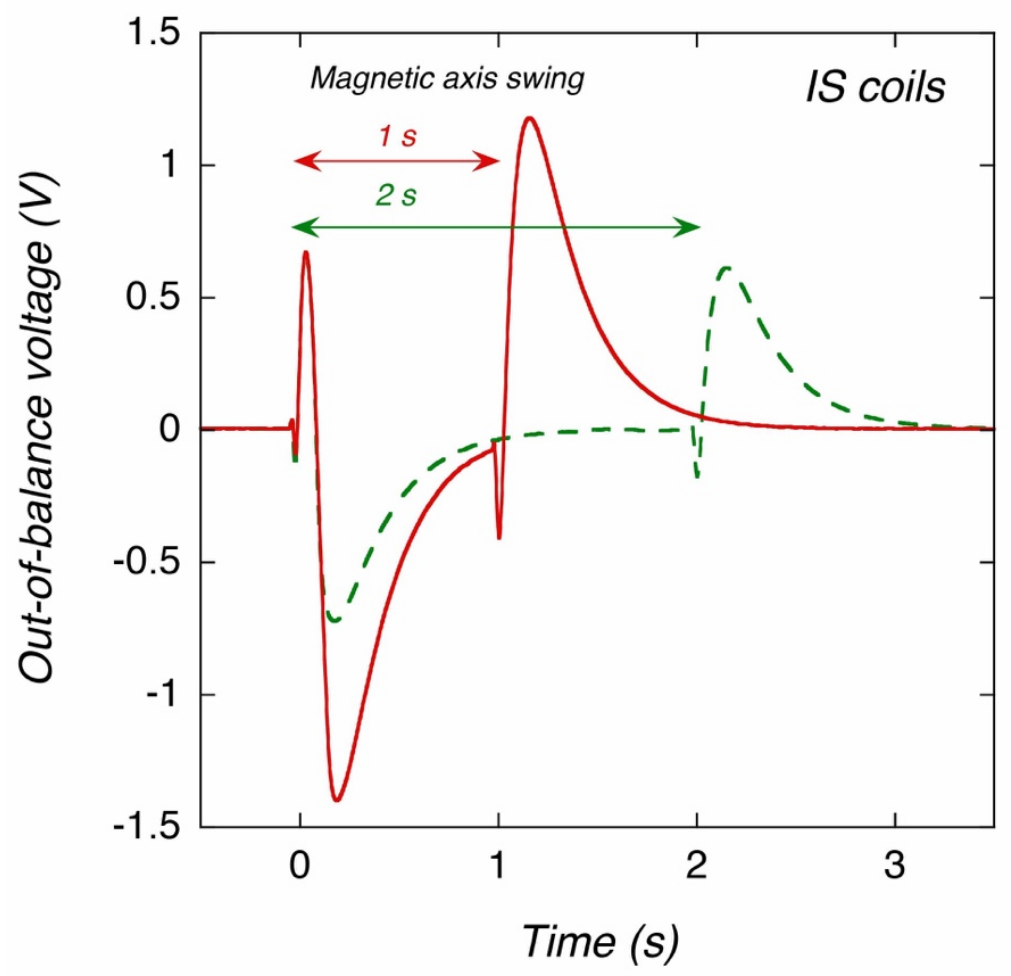

Fig. 9. Out-of-balance voltage from the IS coils during magnetic axis swings for $1 \mathrm{~s}$ (solid line) and $2 \mathrm{~s}$ (dashed line) without plasma.

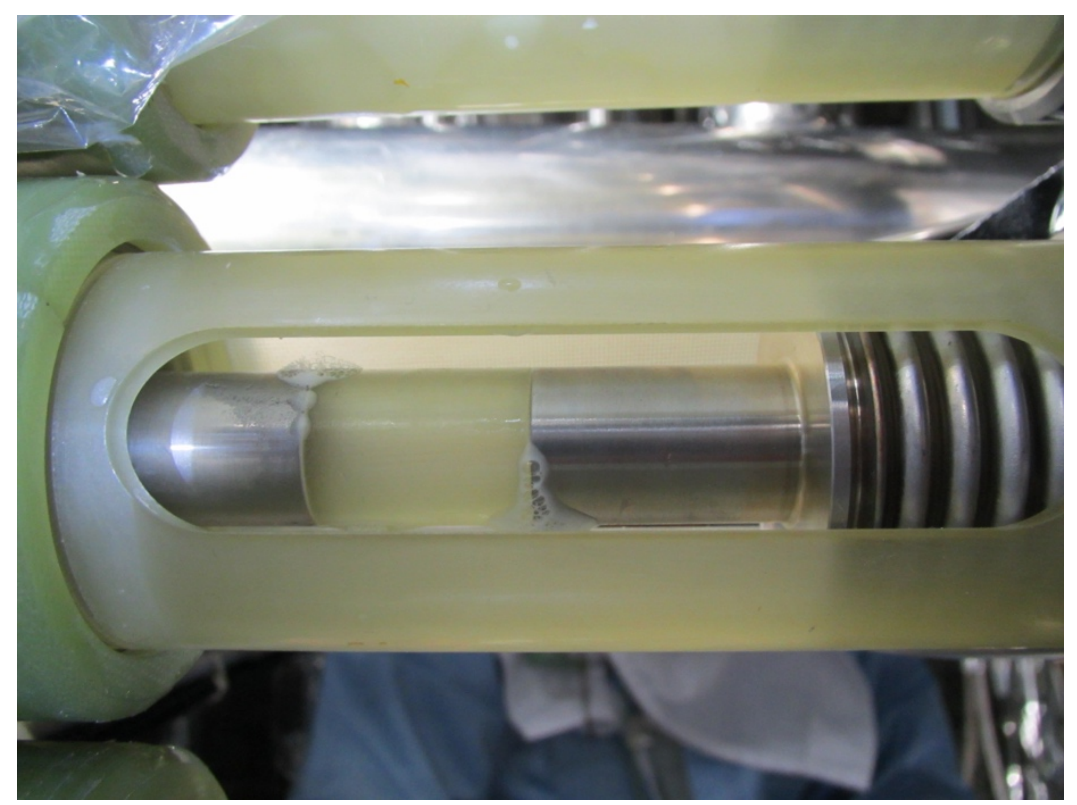

Fig. 10. Soap bubble testing for the cryogenic insulation break after a helium leak. Bubbles are evident at both ends of the FRP pipe. 


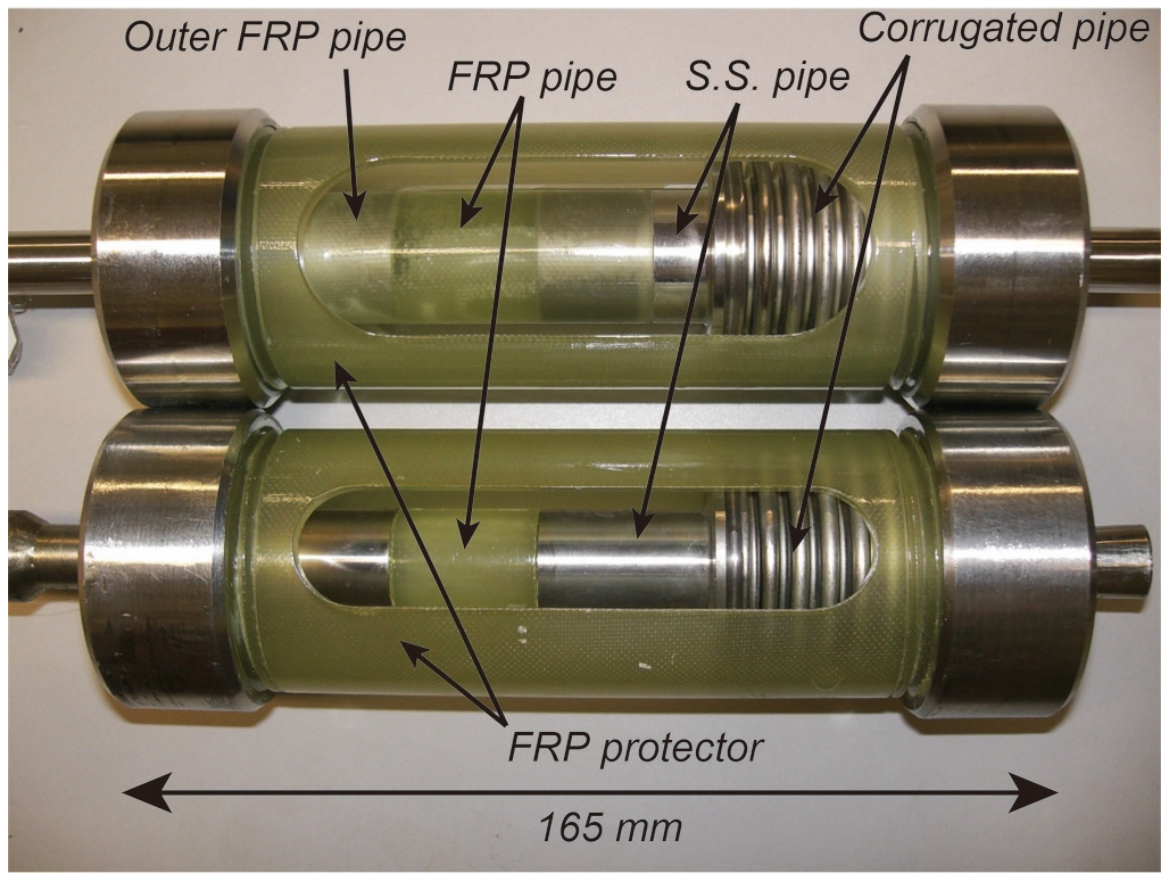

Fig. 11. New improved (upper) and former (lower) cryogenic insulation breaks. The new break has an additional outer FRP pipe. 